\title{
ESTIMACIÓN DE PROPIEDADES DINÁMICAS DE ARCILLAS
}

\author{
Claudia M. González Blandón ${ }^{(1)}$ y Miguel P. Romo Organista ${ }^{(1)}$
}

\begin{abstract}
RESUMEN
Se propone un procedimiento de modelación tipo Masing, para estimar los comportamientos no lineales de propiedades dinámicas de arcillas normalmente consolidadas. Estos comportamientos corresponden a las variaciones del módulo de rigidez al corte y de la relación de amortiguamiento respecto a la deformación angular. El procedimiento es producto de un análisis minucioso de las propuestas realizadas por Romo $(1990,1995)$ y Flores et al. (1999). Dicho análisis sustenta la necesidad de definir valores de la deformación de referencia y del parámetro $B$, para cada una de las propiedades dinámicas. Por ende, se puede concluir que la modelación del módulo de rigidez y de la relación de amortiguamiento, se debe realizar de manera independiente. Finalmente, se ilustra el procedimiento de modelación mediante un ejercicio de aplicación, en el que se obtienen las curvas $G-\gamma$ y $\lambda-\gamma$ de una muestra de arcilla de la Ciudad de México.
\end{abstract}

Palabras clave: Masing; arcilla; índice de plasticidad; módulo de rigidez al corte; relación de amortiguamiento

\begin{abstract}
A modeling procedure based on Masing rules is proposed to estimate the nonlinear dynamic behavior of normally consolidated clays. The properties we are concerned in this paper are the shear modulus and the damping ratio. The procedure is the product of a careful analysis of the models proposed by Romo $(1990,1995)$ and Flores et al. (1999). This analysis supports the need to define values of the reference strain and of the parameter $B$, for each one of the dynamic properties. Therefore, it is concluded that the modeling of shear modulus and damping ratio should be carried out independently. Finally, the modeling procedure through an application exercise is presented. In this exercise, the $G-\gamma$ and $\lambda-\gamma$ curves of a clay sample from Mexico City are obtained.
\end{abstract}

Key words: Masing; clay; plasticity index; shear modulus; damping ratio

\footnotetext{
Artículo recibido el 31 de agosto de 2010 y aprobado para su publicación el 5 de febrero de 2011.

(1) Instituto de Ingeniería, UNAM, Coordinación de Geotecnia. Circuito Escolar, Ciudad Universitaria, Coyoacán, 04510, México D.F., cgonzalezb@ii.unam.mx , mromo@pumas.iingen.unam.mx
} 


\section{INTRODUCCIÓN}

Para evaluar la respuesta dinámica de un depósito de suelo y de sistemas suelo-estructura, es necesario conocer propiedades del material en cuestión, como el módulo de rigidez al esfuerzo cortante, $G$, y la relación de amortiguamiento, $\lambda$. Estas propiedades presentan comportamientos no lineales respecto a la deformación angular, $\gamma$. La obtención experimental de estas propiedades, frecuentemente, es afectada por la disponibilidad de equipos necesarios y los costos de las pruebas requeridas. Por tal razón, se recurre a procesos de modelación que requieren un mínimo de pruebas experimentales y facilitan los análisis.

A la fecha existen modelos (Seed e Idriss, 1970; Hardin y Drnevich, 1972; Ramberg-Osgood, 1970 (descrito por Anderson y Richart, 1976); Romo, 1995; Flores et al., 1999) que consideran, según el tipo de suelo, tendencias en los comportamientos de las propiedades dinámicas. Por ejemplo, en arcilla de la Ciudad de México, para niveles de deformación angular menores o iguales a $10^{-4} \%$, el $G$ y la $\lambda$ permanecen prácticamente constantes; por consiguiente, para $\gamma$ iguales o inferiores a $10^{-4} \%$, se asume que los valores del $G$ y de la $\lambda$ corresponden a las magnitudes máxima del módulo de rigidez al corte, $G_{\max }, \mathrm{y}$ mínima de la relación de amortiguamiento crítico, $\lambda_{\min }$. Para deformaciones angulares superiores a este umbral ( $\gamma=10^{-4} \%$ ), $G$ disminuye hasta un valor mínimo, $G_{\min }$, cercano a cero, y $\lambda$ aumenta hasta llegar a un valor máximo, $\lambda_{\text {máx }}$, prácticamente constante.

Las expresiones propuestas por Romo $(1990,1995)$ y Flores et al. (1999) para la modelación del módulo de rígidez al esfuerzo cortante y la relación de amortiguamiento de suelos son, hasta la fecha, utilizadas para evaluar el comportamiento dinámico de diversos materiales (e.g. González et al., 2007; Ossa y Romo, 2008). Este documento presenta un análisis paramétrico de dichas expresiones, considerando las hipótesis adoptadas en el planteamiento de las mismas. Finalmente, se propone un modelo modificado tipo Masing para obtener comportamientos no lineales del $G$ y de la $\lambda$ de arcillas normalmente consolidadas.

\section{MODELO TIPO MASING}

La modelación de las propiedades dinámicas de un suelo, debe considerar una ley constitutiva que defina el comportamiento elástico (rango de pequeñas deformaciones) y elasto-plástico (rango de grandes deformaciones) de los suelos, tal como lo hace la conocida Regla de Masing (Ishihara, 1996).

Manteniendo dicha regla, Romo $(1990,1995)$ y Flores et al. (1999) propusieron un Modelo tipo Masing, para modelar las relaciones no lineales $G-\gamma$ y $\lambda-\gamma$, cuyas hipótesis son:

- El material sometido a carga armónica con amplitud constante responde de manera estable, si el amortiguamiento histerético es suficiente para atenuar en pocos ciclos la parte transitoria.

- La no linealidad del material es de naturaleza plástica, es decir, la rigidez del suelo regresa a su valor máximo cada vez que la carga cambia de sentido.

- El amortiguamiento es de tipo histerético.

Las expresiones que estos autores propusieron son: 
$G=\left(G_{\text {min }}-G_{\text {máx }}\right) \cdot H(\gamma)+G_{\text {máx }}$

$\lambda=\left(\lambda_{\text {máx }}-\lambda_{\text {min }}\right) \cdot H(\gamma)+\lambda_{\text {min }}$

$H(\gamma)=\left[\frac{\left(\frac{\gamma}{\gamma_{r}}\right)^{2 B}}{1+\left(\frac{\gamma}{\gamma_{r}}\right)^{2 B}}\right]^{A}$

donde: $G_{\operatorname{máx}}$ y $\lambda_{\min }$ corresponden a los valores del $G$ y de la $\lambda$ para el rango de deformaciones, en el cual el comportamiento del suelo es elástico (figura 1); $G_{\text {min }}$ y $\lambda_{\text {máx }}$ son los valores del $G$ y de la $\lambda$, mínimo y máximo respectivamente, alcanzados por el suelo antes de llegar a la falla bajo carga dinámica (figura 1); $\gamma_{r}$ es una deformación de referencia, correspondiente al 50\% de degradación del $G$ (figura 1); $A$ y $B$ son parámetros del suelo que definen la geometría de las curvas $G-\gamma$ y $\lambda-\gamma$ y dependen principalmente del índice de plasticidad, $I P$.

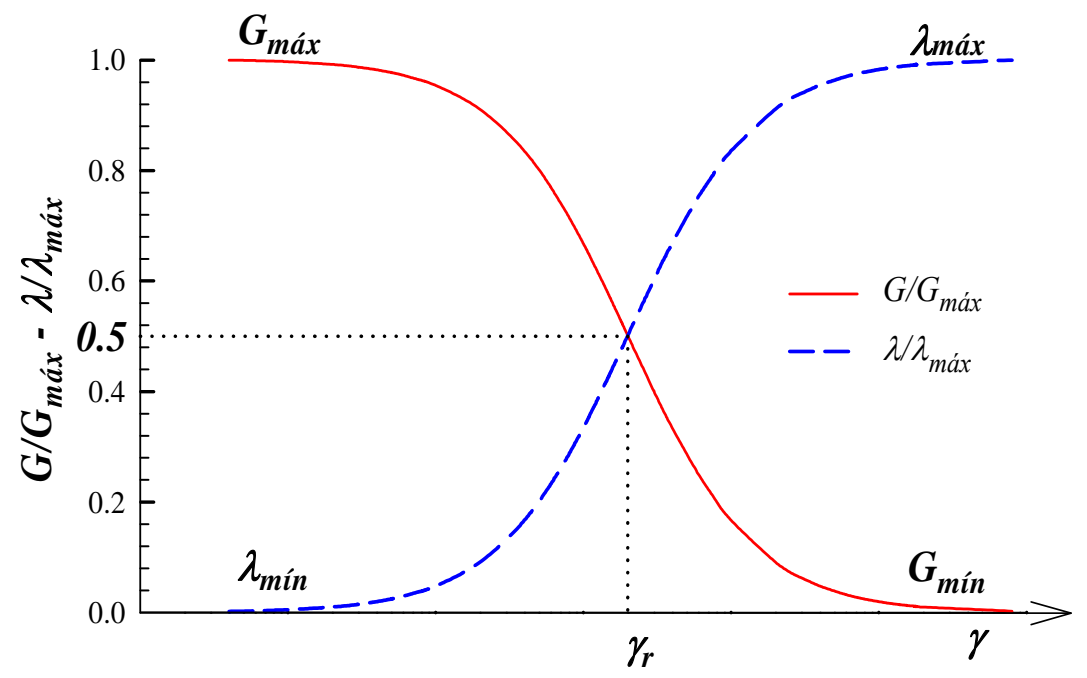

Figura 1. Parámetros empleados por el Modelo tipo Masing.

Las condiciones de frontera (valores extremos) establecidas en las expresiones anteriores, satisfacen los siguientes límites de comportamiento de un suelo:

- Cuando $\gamma$ tiende a infinito: $H(\gamma)$ tiende a uno, esto implica que $G$ tienda a $G_{\min } \mathrm{y} \lambda$ tienda a $\lambda_{\operatorname{máx}}$.

- Cuando $\gamma$ tiende a cero: $H(\gamma)$ tiende a cero, esto conduce a que $G$ tienda a $G_{\text {máx }}$ y $\lambda$ tienda a $\lambda_{\min }$. 


\section{ANÁLISIS DE LOS PARÁMETROS DEL MODELO TIPO MASING}

A continuación se presenta un análisis paramétrico del Modelo tipo Masing.

\section{Parámetros $A$ y $B$}

Los valores de los parámetros $A$ y $B$ son primordiales en los análisis de amplificación dinámica e interacción suelo-estructura; además, son éstos quienes dan la forma particular de las curvas módulo de rigidez versus deformación angular y relación de amortiguamiento crítico versus deformación angular.

\section{Parámetro A}

Para obtener el valor de $A$ basta con manipular la ecuación 1, tomando en cuenta que al $50 \%$ de degradación del módulo de rígidez al esfuerzo cortante, $G_{50}$, la deformación angular $\gamma$ corresponde a $\gamma_{r}$. Entonces,

$G_{50}=\frac{G_{\text {máx }}-G_{\text {min }}}{2}+G_{\text {min }}=\frac{G_{\text {máx }}+G_{\text {min }}}{2}$

Igualando $G_{50}$ (expresión anterior) y la ecuación 1, se tiene

$\frac{G_{\text {máx }}+G_{\text {mín }}}{2}=\left(G_{\text {min }}-G_{\text {máx }}\right) \cdot H(\gamma)+G_{\text {máx }}$

Despejando la función $H(\gamma)$ de la expresión anterior, combinando la ecuación 3 y tomando $\gamma=\gamma_{r}$, se tiene

$\frac{G_{\text {min }}-G_{\text {máx }}}{2\left(G_{\text {min }}-G_{\text {máx }}\right)}=\left[\frac{\left(\frac{\gamma}{\gamma_{r}}\right)^{2 B}}{1+\left(\frac{\gamma}{\gamma_{r}}\right)^{2 B}}\right]^{A}$

$0.5=\left[\frac{1^{2 B}}{1+1^{2 B}}\right]^{A}$

$\log (0.5)=A \cdot \log (0.5)$

$A=1$

Finalmente, al obtener el valor de $A$ constante, se demuestra que este parámetro es independiente del índice de plasticidad. Este hecho difiere del planteamiento del Modelo tipo Masing. Cabe mencionar que aplicando un procedimiento algebraico similar con la relación de amortiguamiento crítico (ecuación 2), se obtiene el mismo resultado $(\boldsymbol{A}=\mathbf{1})$. 


\section{Parámetro B}

Conocido el valor de $A$ es posible despejar $B$ de la ecuación 7, llegando a

$1^{2 B}=1$

De la expresión anterior se concluye que $\boldsymbol{B}$ puede tomar diversos valores. Esta variabilidad de $B$ puede atribuirse, posiblemente, a la estructura característica del material modelado. Para el caso de las arcillas, $B$ dependerá del índice de plasticidad, $I P$.

\section{Deformación $\gamma_{r}$}

La deformación $\gamma_{r}$ (definida de manera arbitraria) representa la deformación correspondiente al punto de inflexión de la curva $G / G_{\text {máx }}-\gamma$ y por consiguiente, a los cambios de curvatura de las relaciones no lineales $G-\gamma$ y $\lambda-\gamma$ (figura 2a).

Para comprobar de manera analítica que $\gamma_{r}$ corresponde al punto de inflexión de las relaciones $G-\gamma$ y $\lambda-\gamma$, se emplea el criterio de la segunda derivada. Previamente, es necesario realizar un mapeo o transformación de abscisas, ya que dicho punto es visible en escala semi-logarítmica y no en escala aritmética (figura 2b).

El mapeo se realiza empleando la siguiente expresión

$t=\left[\log _{10}(x)-n\right] \cdot a$

donde $t$ son los valores transformados de las abscisas, $n$ es el exponente menor del rango de deformaciones manejado en escala logarítmica, $a$ es el número de unidades por ciclo y $x$ es la relación entre deformaciones $\gamma / \gamma_{r}$.

Despejando $x$ de la expresión anterior se obtiene

$x=10^{\frac{t}{a}+n}$

Para aplicar el criterio de la segunda derivada, es preciso partir de la ecuación 1 normalizada y expresada en función de $x$.

$\frac{G}{G_{\text {máx }}}=G_{N}=\left(\frac{G_{\min }}{G_{\text {máx }}}-1\right) \cdot \frac{x^{2 A B}}{\left(1+x^{2 B}\right)^{A}}+1$

Haciendo uso de la regla de la cadena, para obtener la primera derivada de la ecuación anterior, se tiene que

$\frac{d G_{N}}{d t}=\frac{d G_{N}}{d x} \cdot \frac{d x}{d t}=\left(\frac{G_{\min }}{G_{\max }}-1\right) \cdot\left(\frac{\ln (10)}{a}\right) \cdot\left(\frac{2 A B x^{2 A B}}{\left(1+x^{2 B}\right)^{A+1}}\right)$ 


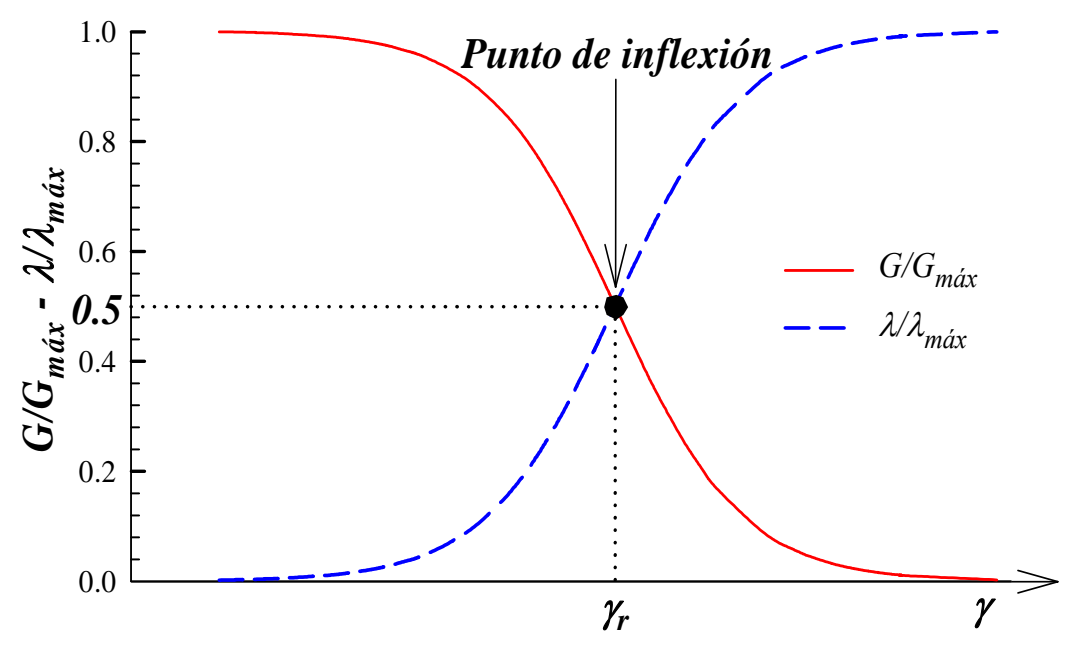

a) Escala semi-logarítmica.

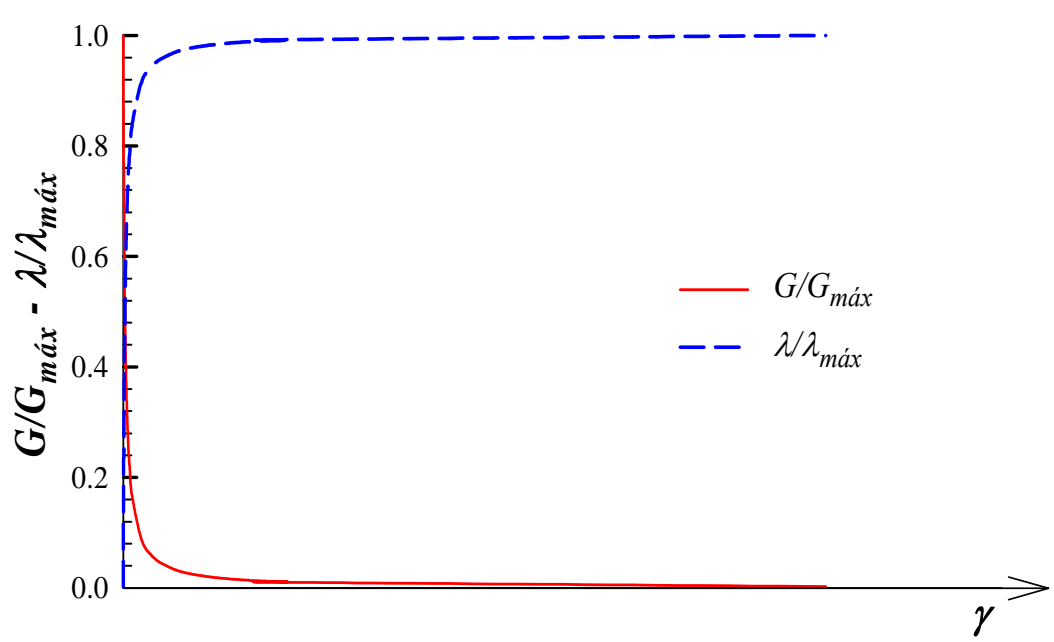

b) Escala aritmética.

Figura 2. Representación gráfica de la funciones normalizadas $G=f(\gamma)$ y $\lambda=f(\gamma)$.

Empleando nuevamente la regla de la cadena, se obtiene que la segunda derivada de la ecuación 13 es igual a

$\frac{d^{2} G_{N}}{d t^{2}}=\frac{d\left(\frac{d G_{N}}{d t}\right)}{d x} \cdot \frac{d x}{d t}=\left(\frac{G_{\text {min }}}{G_{\text {máx }}}-1\right) \cdot\left(\frac{\ln ^{2}(10)}{a^{2}}\right) \cdot\left(\frac{4 A B^{2} x^{2 A B}\left(A-x^{2 B}\right)}{\left(1+x^{2 B}\right)^{A+2}}\right)$

Igualando la expresión 15 a cero y despejando el valor de x, se tiene que

$x=\frac{\gamma}{\gamma_{r}}=A^{\frac{1}{2 B}}$

Conociendo que $A=1$ y por ende $x=1$, se llega a $\gamma=\gamma_{r}$. Ésto demuestra que el punto de inflexión de la curva normalizada del módulo de rigidez, está definido por las coordenadas $\left(\gamma_{r},\left(G_{\text {máx }}+G_{\text {min }}\right) /\left(2 G_{\text {máx }}\right)\right)$ 
y es independiente del valor del parámetro $B$. Cabe mencionar que para la relación de amortiguamiento normalizada $\left(\lambda / \lambda_{\text {máx }}\right)$, el seguir un procedimiento análogo al expuesto, arroja un resultado idéntico.

Demostrada la definición matemática de la deformación $\gamma_{r}$, es evidente que el Modelo tipo Masing considera un único punto de inflexión (obtenido de la relación $G / G_{\text {máx }}-\gamma$ ) para las funciones $G=f(\gamma)$ y $\lambda=f(\gamma)$.

Sin embargo, en resultados experimentales se ha observado que los puntos de inflexión de las relaciones $G-\gamma$ y $\lambda-\gamma$ no coinciden. Normalmente, el cambio de curvatura de la relación de amortiguamiento se presenta en una deformación angular mayor que la deformación correspondiente al punto de inflexión de la relación $G-\gamma$ (figura 3). Por lo tanto, se considera conveniente definir la deformación de referencia $\gamma_{r}$, tanto para la relación $G-\gamma, \gamma_{r G}$, como para la relación $\lambda-\gamma, \gamma_{r \lambda}$.

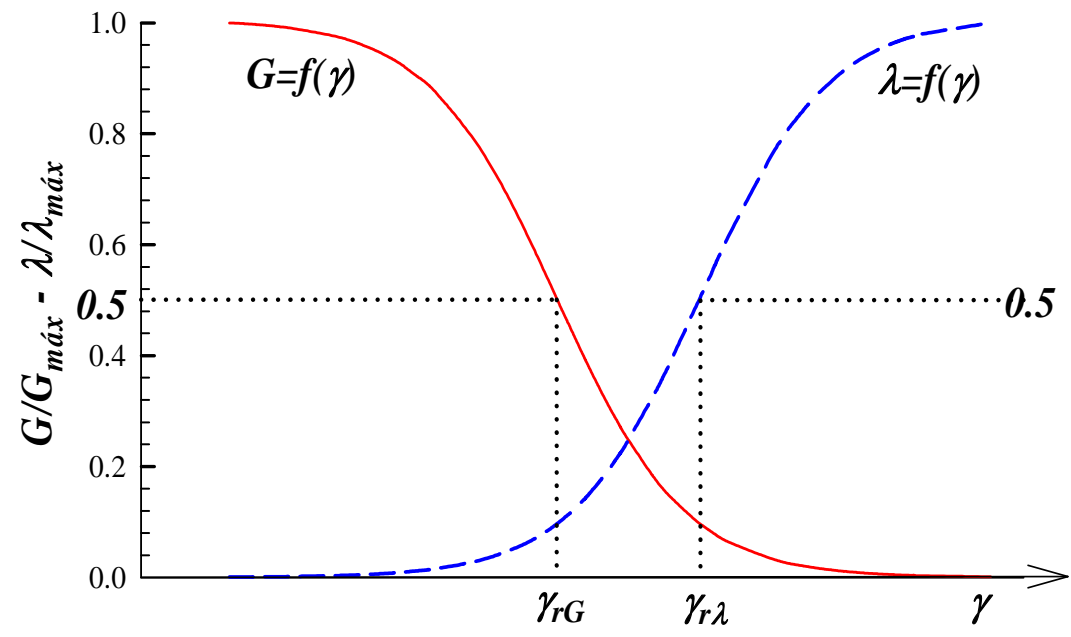

Figura 3. Puntos de inflexión de las relaciones $G-\gamma$ y $\lambda-\gamma$.

Valores $G_{m a ́ x}, G_{m i ́ n}, \lambda_{\text {máx }}$ y $\lambda_{\text {mín }}$

Los valores máximo y mínimo de las propiedades dinámicas $G$ y $\lambda$ se definen experimentalmente y por consiguiente, son característicos y únicos del suelo que se esté modelando.

\section{Módulo de rigidez al esfuerzo cortante}

El empleo del Modelo tipo Masing parte de la función normalizada $G / G_{\text {máx }}=f(\gamma)$; por consiguiente, el efecto del valor máximo del $G$ no se considera. Aunque, es de notar que tanto el $G_{\operatorname{máx}}$ como el $G_{\min }$ corresponden a valores frontera dentro del proceso de modelación. Estos valores no afectan a los demás parámetros empleados por el Modelo (figura 4).

Resultados de investigaciones experimentales concluyen que $G_{\text {máx }}$ depende fuertemente del esfuerzo efectivo de confinamiento y que $G_{\min }$, usualmente, presenta valores cercanos a cero (Romo, 1995). 


\section{Relación de amortiquamiento}

De manera similar al módulo de rigidez al cortante, los valores máximo y mínimo de la relación de amortiguamiento corresponden a valores frontera dentro del proceso de modelación. Igualmente, estos valores no influyen en los parámetros $A, B$ y $\gamma_{r}$, empleados en el Modelo (figura 4).

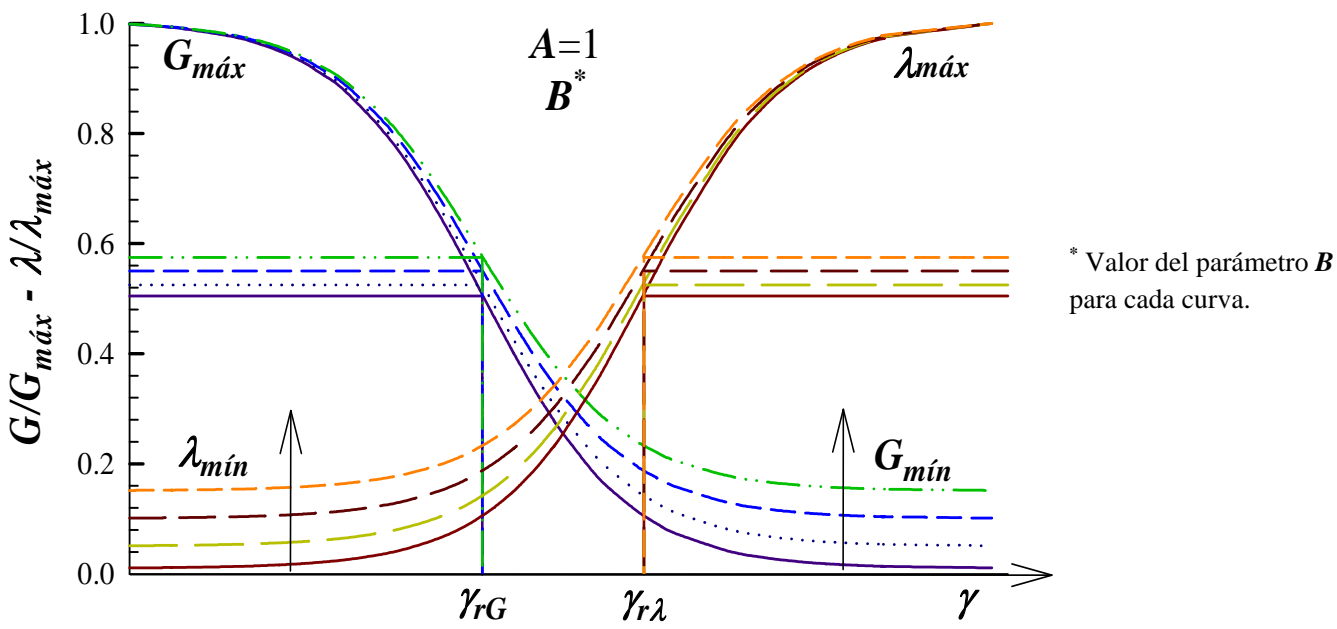

Figura 4. Variación de los valores mínimos de $G$ y $\lambda$, en resultados teóricos.

\section{MODELO MODIFICADO TIPO MASING}

Partiendo del análisis previamente descrito y con el propósito de ampliar la aplicabilidad del Modelo tipo Masing a diversas arcillas, se plantea un procedimiento de modelado nuevo. Este planteamiento conserva tanto las hipótesis como las condiciones de frontera del modelo original.

El Modelo Modificado conserva intacto el proceso de modelación de la relación no lineal $G-\gamma$. Adicionalmente, plantea un procedimiento nuevo para obtener la función $\lambda=f(\gamma)$. Por lo anterior, los procesos de modelación de las propiedades dinámicas son totalmente independientes.

Las expresiones propuestas para el Modelo Modificado tipo Masing son:

$$
\begin{aligned}
& G=\left(G_{\text {mín }}-G_{\text {máx }}\right) \cdot H_{G}+G_{\text {máx }} \\
& \lambda=\left(\lambda_{\text {máx }}-\lambda_{\text {mín }}\right) \cdot H_{\lambda}+\lambda_{\text {mín }} \\
& H_{G}=\frac{\left(\frac{\gamma}{\gamma_{r G}}\right)^{2 B_{G}}}{1+\left(\frac{\gamma}{\gamma_{r G}}\right)^{2 B_{G}}}
\end{aligned}
$$


$H_{\lambda}=\frac{\left(\frac{\gamma}{\gamma_{r \lambda}}\right)^{2 B_{\lambda}}}{1+\left(\frac{\gamma}{\gamma_{r \lambda}}\right)^{2 B_{\lambda}}}$

donde:

$G_{\operatorname{máx}}$ y $\lambda_{\min }$ corresponden a los valores del $G$ y de la $\lambda$ para el rango de deformaciones, en el cual el comportamiento del suelo es elástico (figura 4); estos valores son propios de cada suelo. Para las arcillas estudiadas en esta investigación (tabla 1), $\lambda_{\min }$ presenta valores del orden del 0.5 al 2 por ciento para las arcillas marinas y del 1.5 al 3 por cierto para las arcillas lacustres; en el caso del $G_{\text {máx }}$, se planteó una relación entre dicho valor, el índice de plasticidad, $I P$, y el esfuerzo efectivo de confinamiento, $\sigma_{c}^{\prime}$ (figura 5).

$G_{\min }$ y $\lambda_{\text {máx }}$ son los valores del $G$ y de la $\lambda$, mínimo y máximo respectivamente, alcanzados por el suelo antes de llegar a la falla bajo carga dinámica. Para el caso de las arcillas estudiadas en esta investigación, $G_{\min }$ y $\lambda_{\text {máx }}$ tienden a $3.5 \mathrm{~kg} / \mathrm{cm}^{2}$ y a $17 \%$ para las arcillas marinas y a $1 \mathrm{~kg} / \mathrm{cm}^{2}$ y $14 \%$ para las arcillas lacustres.

$\gamma_{r G}$ y $\gamma_{r \lambda}$ son deformaciones de referencia correspondientes al punto de inflexión de las relaciones experimentales $G-\gamma$ y $\lambda-\gamma$; o sea, deformaciones correspondientes al $50 \%$ de degradación del $G$ $\mathrm{y}$ al $50 \%$ de aumento de la $\lambda$, respectivamente. Estas deformaciones dependen del índice de plasticidad de la arcilla en estudio: a mayor IP mayores serán las deformaciones de referencia (figura 6).

$B_{G}$ y $B_{\lambda}$ son constantes que definen la geometría característica de las curvas $G-\gamma$ y $\lambda-\gamma$, respectivamente. Al igual que las deformaciones de referencia, los valores de las constantes $B_{G} \mathrm{y}$ $B_{\lambda}$ dependen del índice de plasticidad, $I P$, (figura 7).

Tabla 1. Información general de los materiales utilizados.

\begin{tabular}{|c|c|c|}
\hline & \multicolumn{2}{|c|}{ MATERIALES } \\
\hline & $\begin{array}{l}\text { Arcilla lacustre } \\
\text { Ciudad de México }\end{array}$ & $\begin{array}{l}\text { Arcilla marina } \\
\text { Golfo de México }\end{array}$ \\
\hline Localización & Ex Lago de Texcoco & $\begin{array}{c}\text { Complejo Petrolero } \\
\text { Ku-Maloob-Zaap }\end{array}$ \\
\hline Clasificación & $\mathrm{CH}-\mathrm{OH}-\mathrm{MH}$ & $\mathrm{CH}$ \\
\hline Densidad de sólidos & $2.40-2.82$ & $2.66-2.90$ \\
\hline Índice de plasticidad & $135 \%-288 \%$ & $13 \%-61 \%$ \\
\hline Relación de vacíos & $7.20-10.40$ & $1.02-2.25$ \\
\hline Contenido natural de agua & $275 \%-399 \%$ & $26 \%-78 \%$ \\
\hline Pruebas en Columna Resonante & 6 & 21 \\
\hline Pruebas en Cámara Triaxial Cíclica & 6 & 18 \\
\hline
\end{tabular}




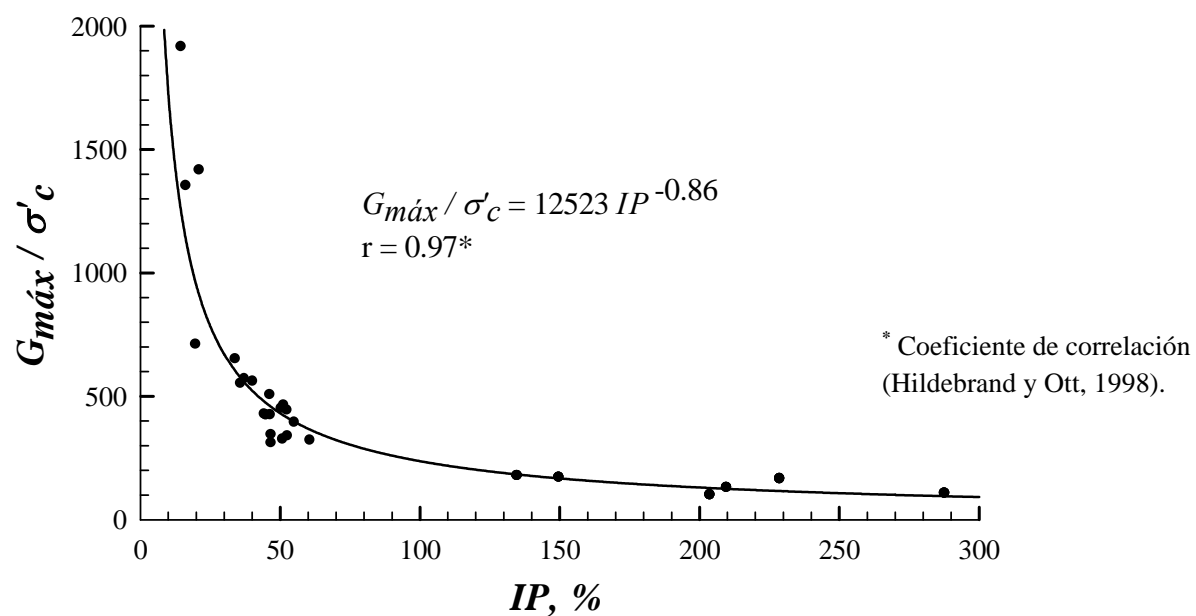

Figura 5. Variación de la relación $G_{m a ́ x} / \sigma_{c}^{\prime}$ respecto al $I P$.

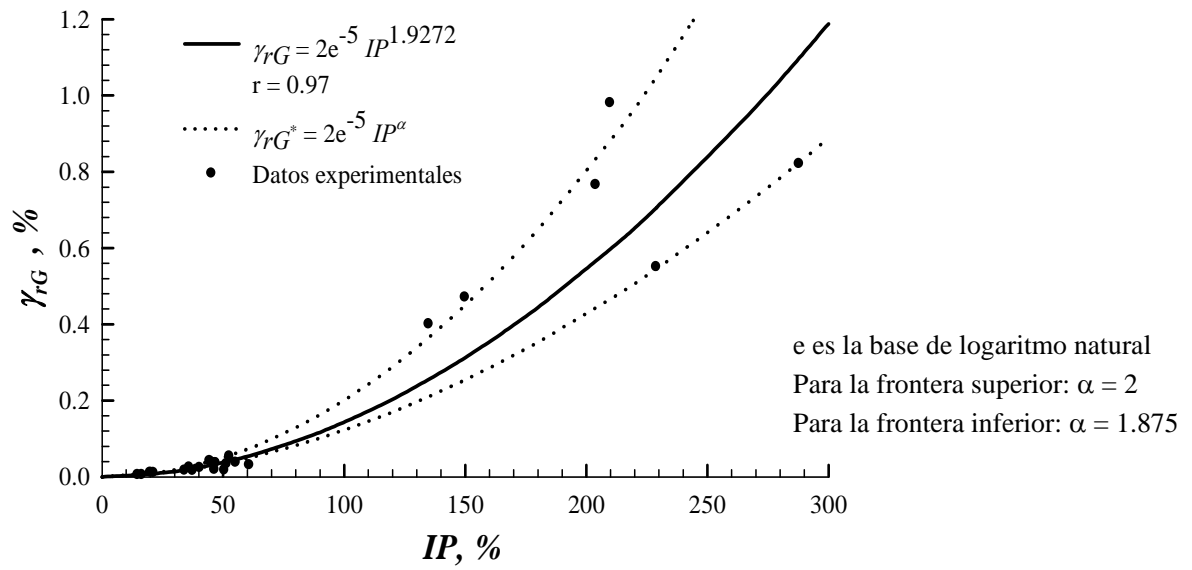

a)

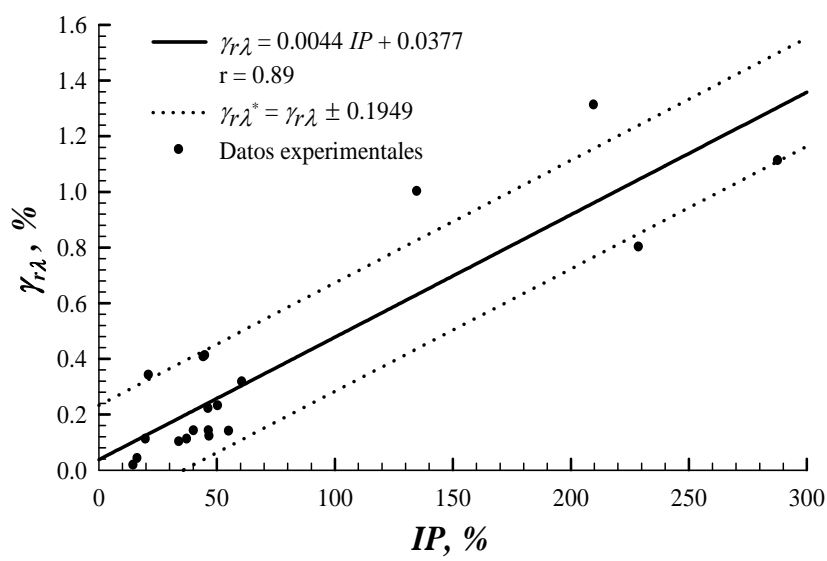

b)

Figura 6. Variación de $\gamma_{r G}$ y $\gamma_{r \lambda}$ respecto al $I P$. 


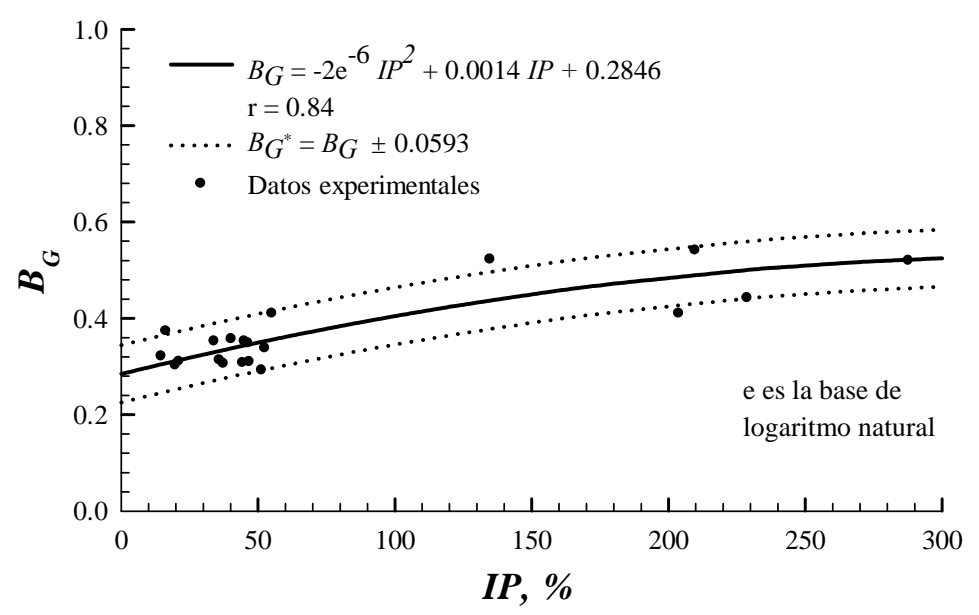

a)

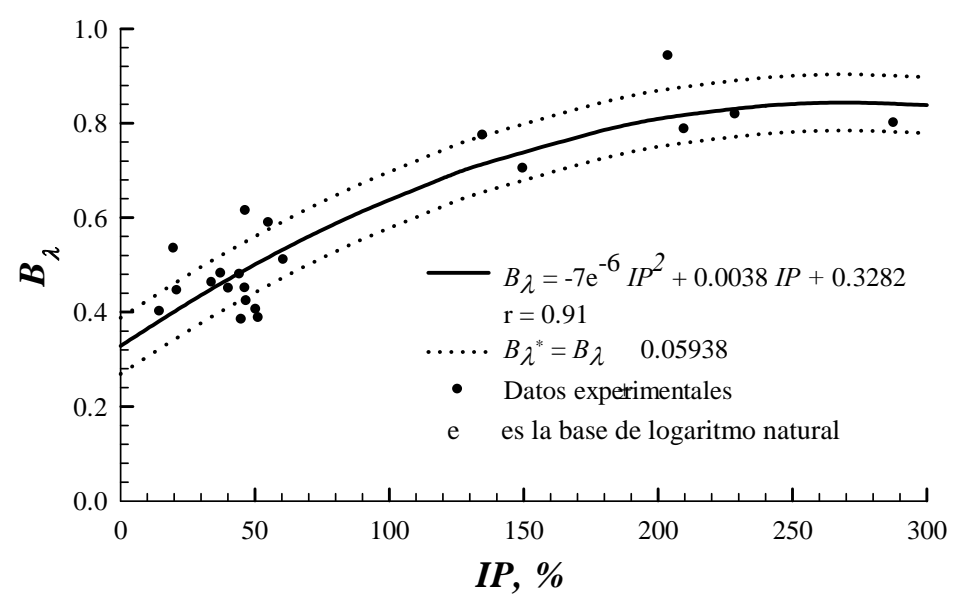

b)

Figura 7. Variación de $B_{G}$ y $B_{\lambda}$ respecto al $I P$.

El Modelo Modificado corresponde a un procedimiento de estimación de las propiedades dinámicas de arcillas normalmente consolidadas. Para este caso, los parámetros $B$ y $\gamma_{r}$ son función del índice de plasticidad. Además, cabe destacar que este Modelo Modificado es aplicable a todo material con comportamiento elasto-plástico (Regla de Masing), donde los parámetros $B$ y $\gamma_{r}$ sean funciones de propiedades preponderantes en la estructura característica del material en estudio (e.g. materiales granulares, con parámetros $B$ y $\gamma_{r}$ como funciones de la consistencia relativa).

\section{VALIDACIÓN DEL MODELO MODIFICADO}

Para evaluar la confiabilidad del Modelo Modificado se presentan resultados experimentales y teóricos de las propiedades dinámicas de dos materiales. Estos materiales presentan procesos de formación distintos y por ende, diferencias notables de estructura y de índices de plasticidad (tabla 2). Adicionalmente, el anexo contiene un ejercicio de aplicación que también permite validar la efectividad del modelo propuesto. 
Las figuras 8 y 9 muestran algunos de los resultados experimentales, obtenidos de pruebas dinámicas realizadas en columna resonante y en cámara triaxial cíclica. También, presentan resultados teóricos producto de la aplicación del Modelo Modificado. En estas figuras se aprecia la gran proximidad de las estimaciones a los datos experimentales, tanto para el módulo de rigidez al esfuerzo cortante como para la relación de amortiguamiento, de las arcillas en cuestión (tabla 2).

Tabla 2. Materiales empleados en el proceso de validación.

\begin{tabular}{|c|c|c|c|c|}
\hline & \multicolumn{4}{|c|}{ MATERIALES } \\
\hline & \multicolumn{2}{|c|}{$\begin{array}{c}\text { Arcilla lacustre } \\
\text { Ciudad de México }\end{array}$} & \multicolumn{2}{|c|}{$\begin{array}{c}\text { Arcilla marina } \\
\text { Golfo de México }\end{array}$} \\
\hline & I & II & I & II \\
\hline Clasificación & $\mathrm{MH}$ & MH & $\mathrm{CH}$ & $\mathrm{CH}$ \\
\hline Densidad de sólidos & 2.74 & 2.53 & 2.8 & 2.82 \\
\hline Índice de plasticidad, \% & 150 & 135 & 46.6 & 40.4 \\
\hline Relación de vacíos & 8.7 & 6.5 & 1.47 & 1.24 \\
\hline Contenido natural de agua, \% & 311 & 280 & 51.1 & 38 \\
\hline $\begin{array}{l}\text { Esfuerzo efectivo de } \\
\text { confinamiento, } \mathrm{kg} / \mathrm{cm}^{2}\end{array}$ & 0.68 & 1.03 & 1.6 & 1.64 \\
\hline
\end{tabular}

La calidad de un proceso de modelación está directamente ligada a la confiabilidad con la que son determinadas las variables que influyen en dicho proceso, la cual se ve reflejada de cierta manera en el coeficiente de correlación (Hildebrand y Ott, 1998). En esta investigación, con la aplicación del Modelo Modificado, se obtuvieron relaciones teóricas $G-\gamma$ y $\lambda-\gamma$ con coeficientes de correlación, normalmente, superiores a 0.95 y en la gran mayoría valores arriba de 0.99 .

Por consiguiente, se considera que el Modelo Modificado amplía el rango de aplicabilidad (distintas arcillas) y mejora la capacidad de estimación (propiedades dinámicas) del Modelo tipo Masing.

Las figuras 10 y 11 presentan una comparación de las curvas $G-\gamma$ y $\lambda-\gamma$ con resultados experimentales obtenidos en arcillas marinas y arcillas lacustres. En estas figuras se aprecia con mayor claridad el alto grado de predicción del modelo propuesto. Además, se puede verificar que a mayor índice de plasticidad, mayores serán los valores de las deformaciones de referencia empleados en la modelación, $\gamma_{r G}$ y $\gamma_{r \lambda}$.

Los valores de los coeficientes de correlación obtenidos en la modelación son bastante buenos; por lo que se puede argüir que el Modelo Modificado es aplicable a distintas arcillas. Sin embargo, durante la modelación se percibió que a mayor índice de plasticidad, la variabilidad en los coeficientes de correlación aumenta. Esto puede deberse a características mineralógicas, fisicoquímicas y ambiente de depositación del material. 


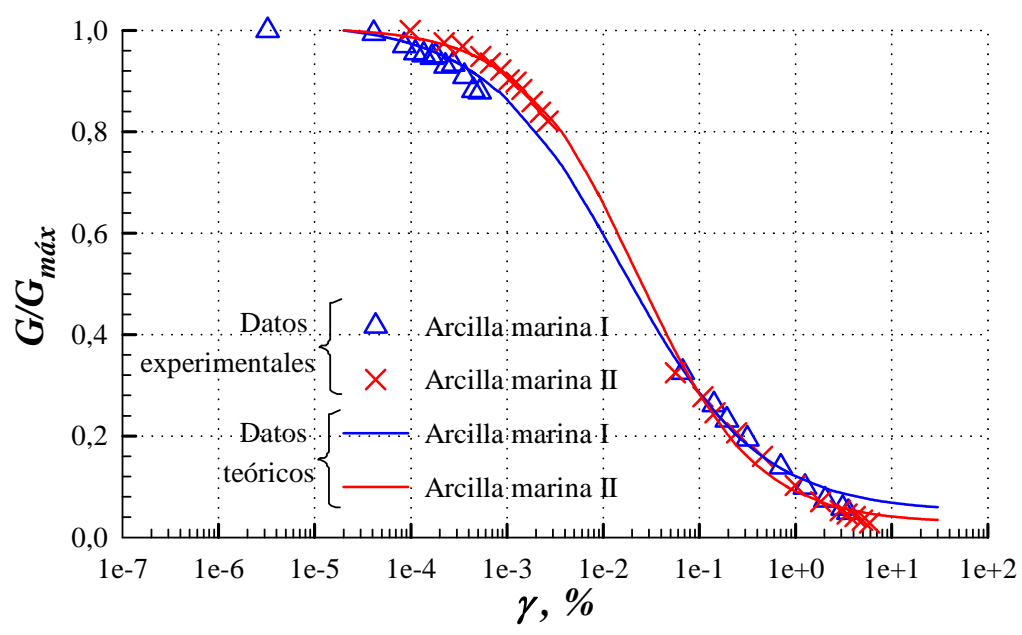

a) Relación no lineal $G-\gamma$.

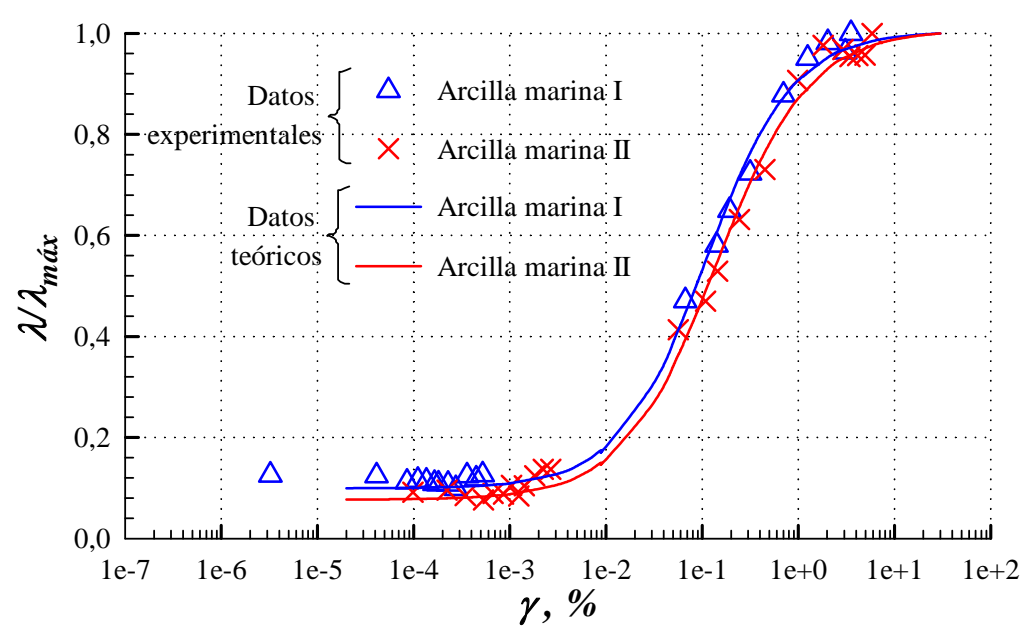

b) Relación no lineal $\lambda-\gamma$.

Figura 8. Resultados experimentales y teóricos de arcillas marinas 


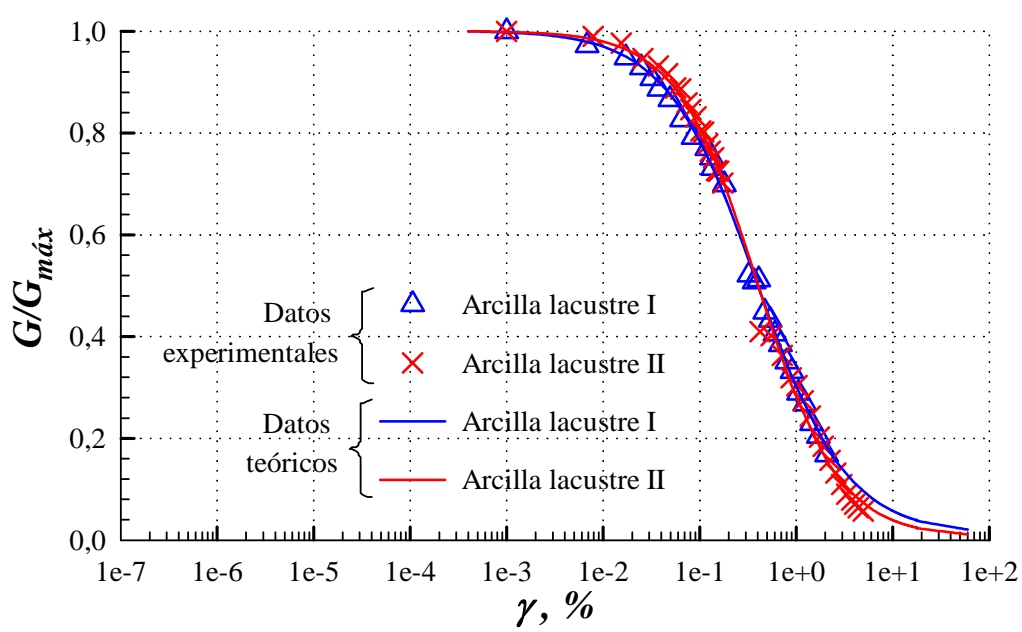

a) Relación no lineal $G-\gamma$.

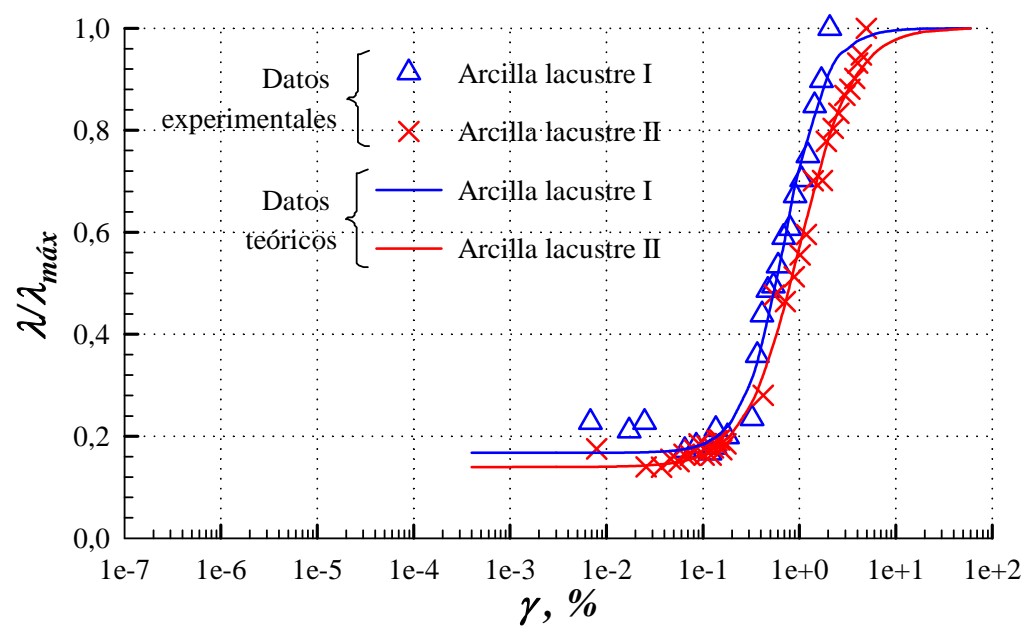

b) Relación no lineal $\lambda-\gamma$.

Figura 9. Resultados experimentales y teóricos de arcillas lacustres. 


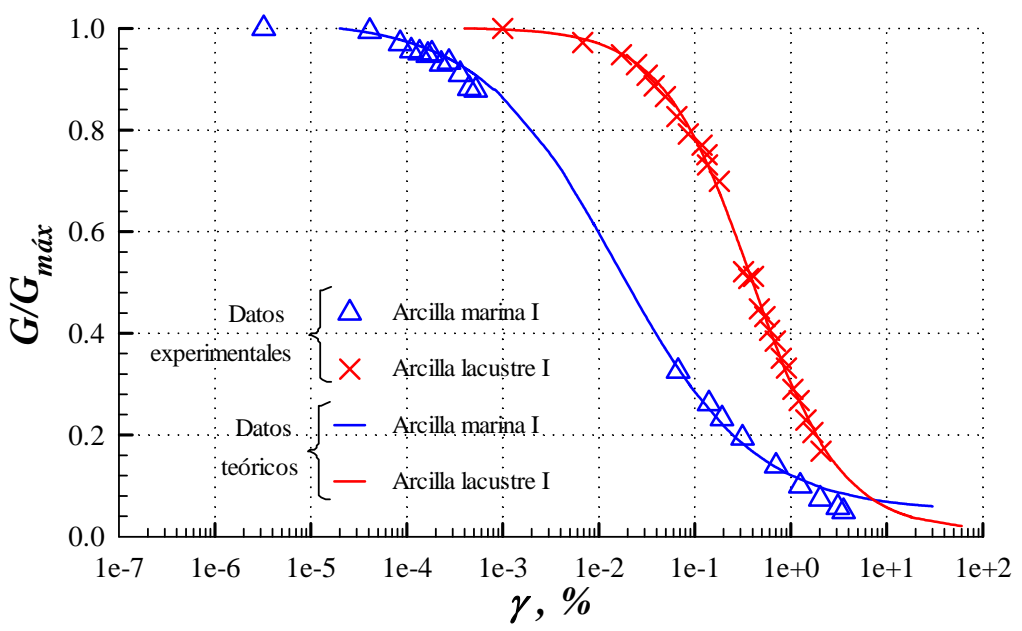

a) Arcillas lacustres tipo I.

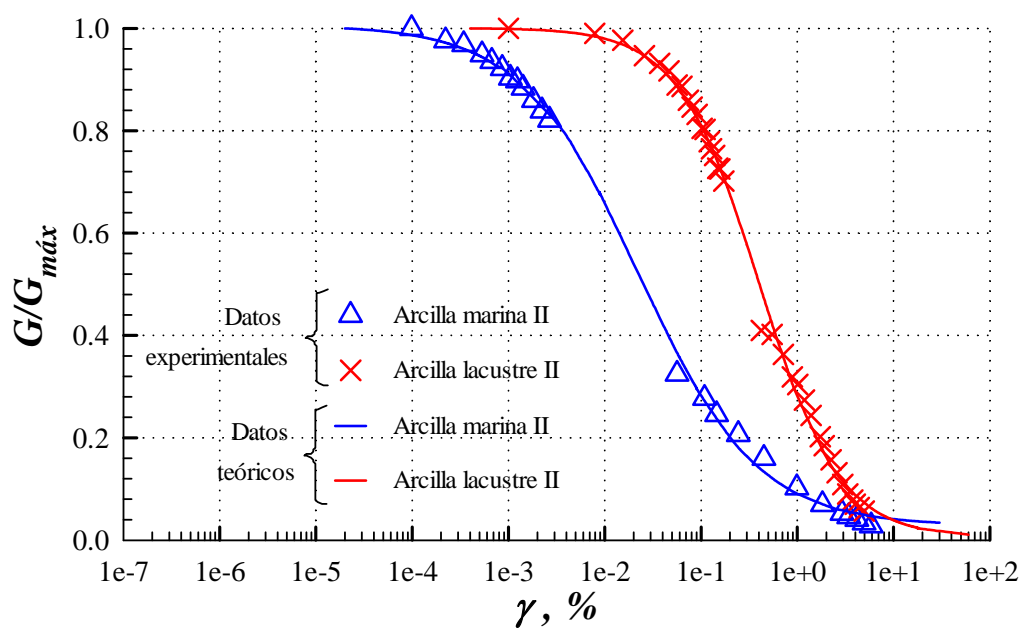

b) Arcillas lacustres tipo II.

Figura 10. Relación no lineal $G-\gamma$ de distintos materiales. 


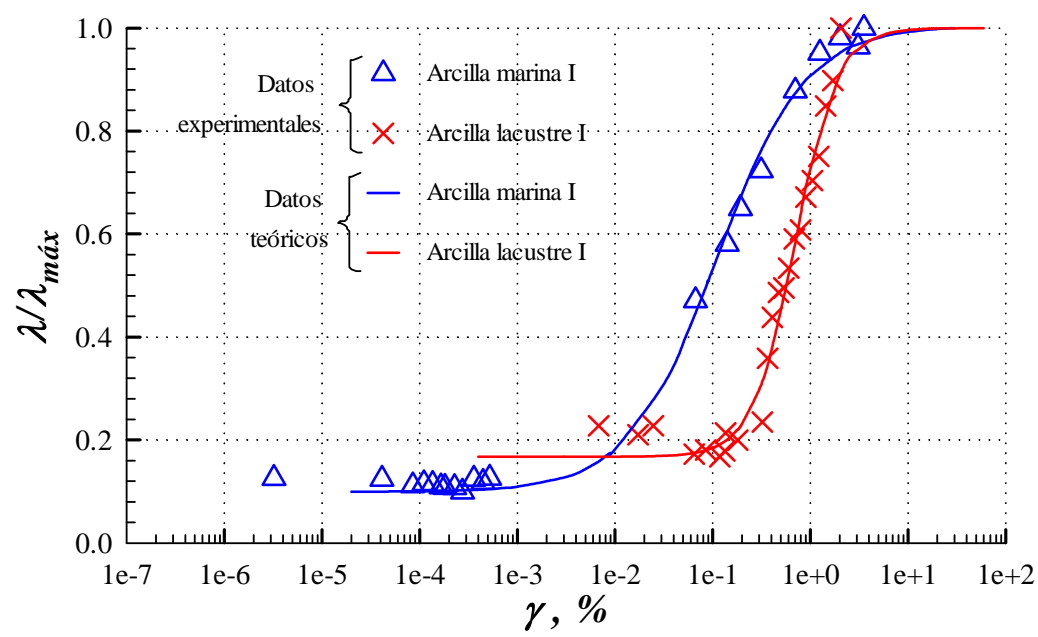

a) Arcillas lacustres tipo I.

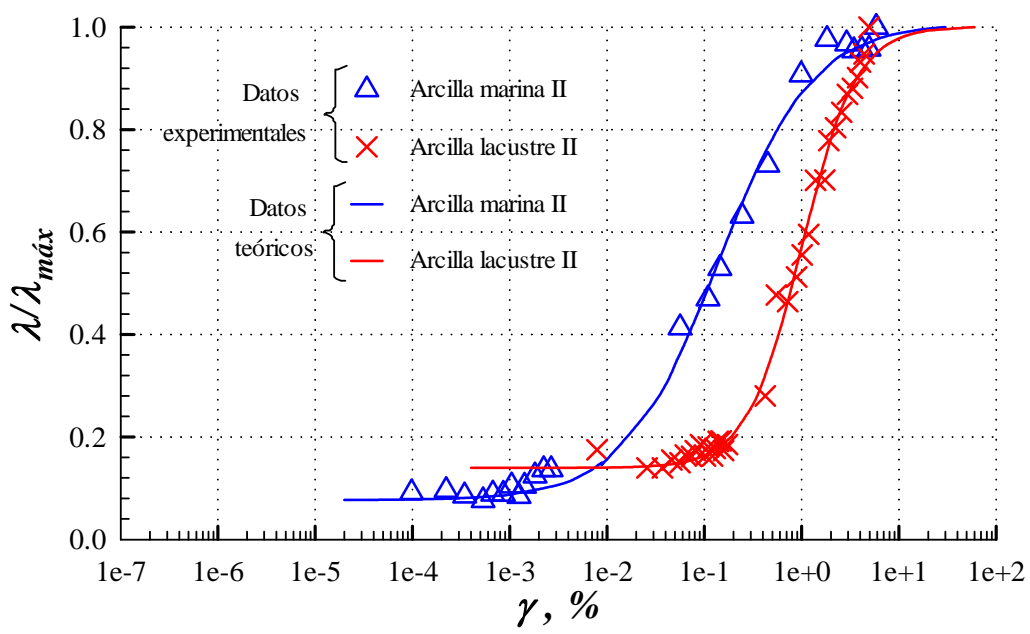

b) Arcillas lacustres tipo II.

Figura 11. Relación no lineal $\lambda-\gamma$ de distintos materiales.

\section{CONCLUSIONES}

A partir de un análisis paramétrico del Modelo tipo Masing y de resultados experimentales, se planteó un Modelo Modificado que permite obtener mejores estimaciones de las propiedades dinámicas de distintas arcillas.

Se demostró que el parámetro $A$ definido en el Modelo tipo Masing corresponde a una constante igual a uno y por lo tanto, es independiente del índice de plasticidad del material a modelar.

Se probó que la deformación de referencia necesaria para obtener la relación teórica $G-\gamma$, difiere de la deformación requerida para obtener la relación $\lambda-\gamma$. Adicionalmente, se demostró que estas 
deformaciones, $\gamma_{r G}$ y $\gamma_{r \lambda}$, corresponden a los puntos de inflexión de las curvas $G-\gamma$ y $\lambda-\gamma$ y a su vez, son independientes del parámetro $B$.

Los parámetros $B_{G}$ y $B_{\lambda}$ definen la geometría particular de las relaciones $G-\gamma$ y $\lambda-\gamma$, respectivamente. Para el caso de las arcillas, estos parámetros son funciones del índice de plasticidad y se pueden obtener empleando correlaciones (e.g. figura 7) o procedimientos matemáticos alternos (e.g. mínimos cuadrados). Esta versatilidad en el cálculo de los parámetros $B_{G} \mathrm{y} B_{\lambda}$, permite que el Modelo Modificado pueda ser aplicado a cualquier tipo de material.

Actualmente, el Modelo Modificado tipo Masing está formulado para estimar propiedades dinámicas exclusivamente de arcillas normalmente consolidadas, donde los parámetros $B$ y $\gamma_{r}$ son funciones del índice de plasticidad. Sin embargo, este Modelo Modificado brinda una plataforma de partida a investigaciones futuras, dedicadas al estudio de las propiedades dinámicas de materiales con comportamiento elasto-plástico, donde los parámetros $B$ y $\gamma_{r}$ no dependan necesariamente del IP (e.g. suelos granulares y polímeros).

\section{AGRADECIMIENTOS}

Los autores agradecen al Dr. Osvaldo Flores por proporcionar los datos experimentales presentados en el Anexo, así como al Instituto de Ingeniería de la UNAM y al Consejo Nacional de Ciencia y Tecnología CONACyT por el apoyo económico otorgado al primer autor. Igualmente, se agradece al Posgrado de Ingeniería de la UNAM por la formación académica brindada al primer autor.

\section{REFERENCIAS}

Anderson, D G y Jr. F E Richart (1976), “Effects of straining on shear modulus of clays”, ASCE Journal of the Geotechnical Engineering Division, Vol. 102, No. GT9, pp. 975-987.

Hildebrand, D K y R. L. Ott (1996), Estadística aplicada a la administración y a la economía, Pearson Prentice Hall, Addison Wesley Iberoamericana S.A., México, Primera reimpresión.

Flores, O, M P Romo, L Vega, B García y R Flores (1999), “Ensayes estáticos y dinámicos de las arcillas del corazón impermeable de la presa revolución mexicana (El Guineo)”, Informe Interno del Instituto de Ingeniería, UNAM, México.

González, C M, O Flores, M P Romo y C Dorado (2007), "Modelación de las propiedades dinámicas de suelos compactados saturados”, XIII Conferencia Panamericana de Mecánica de Suelos e Ingeniería Geotécnica, Isla Margarita, Venezuela.

Hardin, B O y V P Drnevich (1972), "Shear modulus and damping in soils: design equations and curves”, ASCE Journal of the Soil Mechanics and Foundation Division, Vol. 98, No. SM7, pp. 667-692.

Ishihara, K (1996), Soil behavior in earthquake geotechnics, Clarendon Press, Oxford University Press, Great Britain.

Ossa, A y M P Romo (2008), “A model for EPS dynamic shear modulus and damping ratio”, The First Panamerican Geosynthetics Conference, Cancún, México. 
Romo, M P (1990), “Comportamiento dinámico de la arcilla de la Ciudad de México y su repercusión en la ingeniería de cimentaciones”, Simposio El Subsuelo de la Cuenca del Valle de México y su Relación con la Ingeniería de Cimentaciones a Cinco Años del Sismo, pp. 83-94.

Romo, M P (1995), “Clay behavior, ground response and soil-structure interaction studies in México city (state of the art paper)", Third International Conference on Recent Advances in Geotechnical Earthquake Engineering and Soil Dynamics, St. Louis Missouri (USA), Vol. II, pp. 1039-1051.

Seed, H B e I M Idriss (1970), "Soil moduli and damping factors for dynamics response analysis”, Report Earthquake Engineering Research Centre, University of California, Berkeley, USA. 


\section{ANEXO EJERCICIO DE APLICACIÓN}

Se ha solicitado la construcción de una determinada obra en la Ciudad de México, la cual se desplantará sobre un depósito de arcilla. La información de dicho depósito, con la que se cuenta inicialmente, se presenta en la tabla A-1.

Tabla A-1. Información del depósito de suelo.

\begin{tabular}{cccc}
\hline $\begin{array}{c}\text { Clasificación del } \\
\text { material }\end{array}$ & $\begin{array}{c}\text { Densidad de } \\
\text { sólidos }\end{array}$ & $\begin{array}{c}\text { Índice de } \\
\text { plasticidad, \% }\end{array}$ & $\begin{array}{c}\text { Contenido natural } \\
\text { de agua, \% }\end{array}$ \\
\hline $\mathrm{CH}$ & 2.65 & 194 & 370 \\
\hline
\end{tabular}

Para realizar los análisis de respuesta del depósito de suelo y de interacción suelo-estructura necesarios, es preciso conocer las propiedades dinámicas del suelo que integra el depósito: módulo de rigidez al esfuerzo cortante, $G$, relación de amortiguamiento, $\lambda$, y su variación con la deformación angular, $\gamma$. Estos parámetros pueden obtenerse mediante pruebas de laboratorio; sin embargo, por cuestiones de tiempo y disponibilidad de equipo, se decide realizar un procedimiento de estimación dinámica aplicando el Modelo Modificado tipo Masing. Para esto, es indispensable contar con la información de la tabla A-2.

Tabla A-2. Esfuerzo efectivo de confinamiento y valores frontera para el proceso de modelación dinámica.

\begin{tabular}{ccccc}
\hline $\begin{array}{c}\text { Esfuerzo efectivo de } \\
\text { confinamiento } \\
\sigma_{3}^{\prime} \dot{o} \sigma_{c}^{\prime}, \mathrm{kg} / \mathrm{cm}^{2}\end{array}$ & $\begin{array}{c}\text { Valor máximo del } G \\
G_{\text {máx }}, \mathrm{kg} / \mathrm{cm}^{2}\end{array}$ & $\begin{array}{c}\text { Valor mínimo del } G \\
G_{\text {min }}, \mathrm{kg} / \mathrm{cm}^{2}\end{array}$ & $\begin{array}{c}\text { Valor máximo de la } \lambda \\
\lambda_{\text {máx }}, \%\end{array}$ & $\begin{array}{c}\text { Valor mínimo de la } \lambda \\
\lambda_{\text {min }}, \%\end{array}$ \\
\hline 0.68 & 91.77 & 0.50 & 14.00 & 2.5 \\
\hline
\end{tabular}

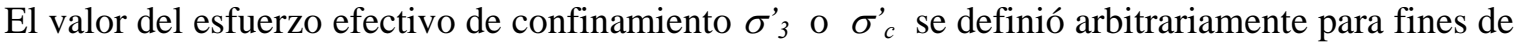
este ejercicio, pero cabe mencionar que este valor, normalmente, corresponde al esfuerzo efectivo de confinamiento en campo para una profundidad determinada. Una vez definido el valor del $\sigma_{c}^{\prime}$ y previo conocimiento del índice de plasticidad, IP (tabla A-1), se puede obtener el valor máximo del módulo de rigidez mediante la expresión A-1 o bien la figura A-1 (correspondiente a la figura 5). Respecto a los valores mínimos $G_{\min }$, $\lambda_{\min }$ y el valor máximo $\lambda_{\operatorname{máx}}$ se tomaron valores experimentales reportados previamente para arcillas de la Ciudad de México (Romo, 1995).

$G_{\text {máx }} / \sigma_{c}^{\prime}=12523 \cdot I P^{-0.86}$

$G_{\text {máx }}=12523 \cdot 194^{-0.86} \cdot 0.68=91.77 \frac{\mathrm{kg}}{\mathrm{cm}^{2}}$

Obtenidos los valores frontera del Modelo Modificado, se prosigue con el cálculo de las deformaciones de referencia $\gamma_{r G}$ y $\gamma_{r \lambda}$, así como de las constantes $B_{G}$ y $B_{\lambda}$, empleando las ecuaciones A-2 a la A-5 o bien las figuras A-2 y A-3 (correspondientes a las figuras 6 y 7). La tabla A-3 agrupa los valores de los parámetros del Modelo. 


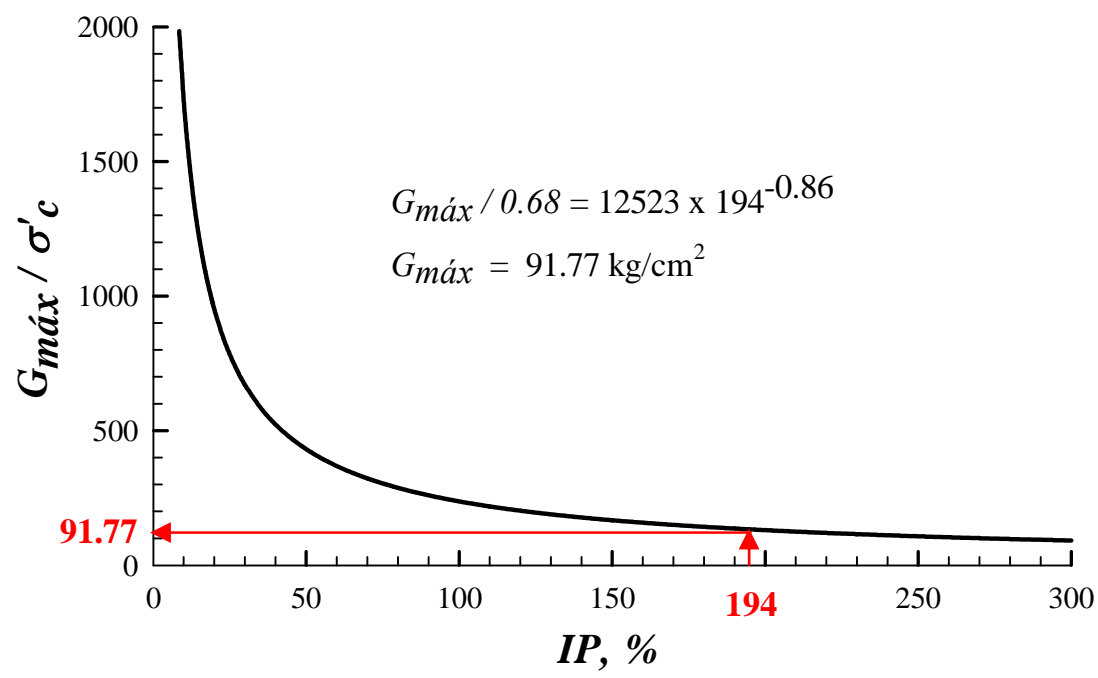

Figura A-1. Obtención del $G_{m a ́ x}$.

$$
\begin{aligned}
& \gamma_{r G}=2 e^{-5} \cdot I P^{1.875} \\
& \gamma_{r G}=2 e^{-5} \cdot 194^{1.875}=0.3896 \\
& \gamma_{r \lambda}=0.0044 \cdot I P+0.0377-0.16 \\
& \gamma_{r \lambda}=0.0044 \cdot 194+0.0377-0.16=0.7313 \\
& B_{G}=-2 e^{-6} \cdot I P^{2}+0.0014 \cdot I P+0.2846 \\
& B_{G}=-2 e^{-6} \cdot 194^{2}+0.0014 \cdot 194+0.2846=0.4809 \\
& B_{\lambda}=-7 e^{-6} \cdot I P^{2}+0.0038 \cdot I P+0.3282+0.05938 \\
& B_{\lambda}=-7 e^{-6} \cdot 194^{2}+0.0038 \cdot 194+0.3282+0.05938=0.8613
\end{aligned}
$$

Tabla A-3. Valores de los parámetros empleados por el Modelo Modificado.

\begin{tabular}{cccc}
\hline$\gamma_{r G}, \%$ & $\gamma_{r \lambda}, \%$ & $B_{G}$ & $B_{\lambda}$ \\
\hline 0.3896 & 0.7313 & 0.4809 & 0.8613 \\
\hline
\end{tabular}

Definidos los valores frontera y los parámetros empleados por el Modelo prosigue asumir un rango amplio de deformaciones angulares (columnas 1 y 6 de la tabla A-4) para calcular las funciones $H_{G}$ y $H_{\lambda}$ (ecuaciones A-6 y A-7, correspondientes a las expresiones 19 y 20, columnas 3 y 8 de la tabla A-4) y posteriormente las propiedades dinámicas $G$ y $\lambda$ (ecuaciones A-8 y A-9, correspondientes a las expresiones 17 y 18, columnas 4 y 9 de la tabla A-4). 


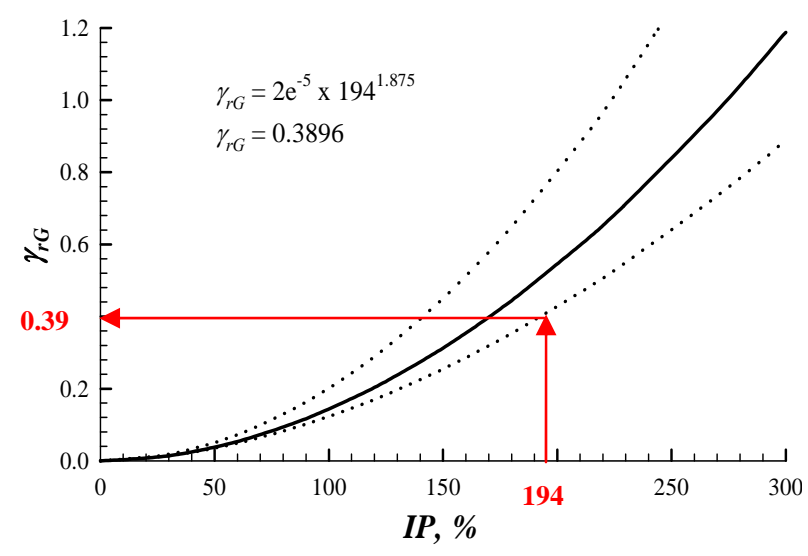

a)

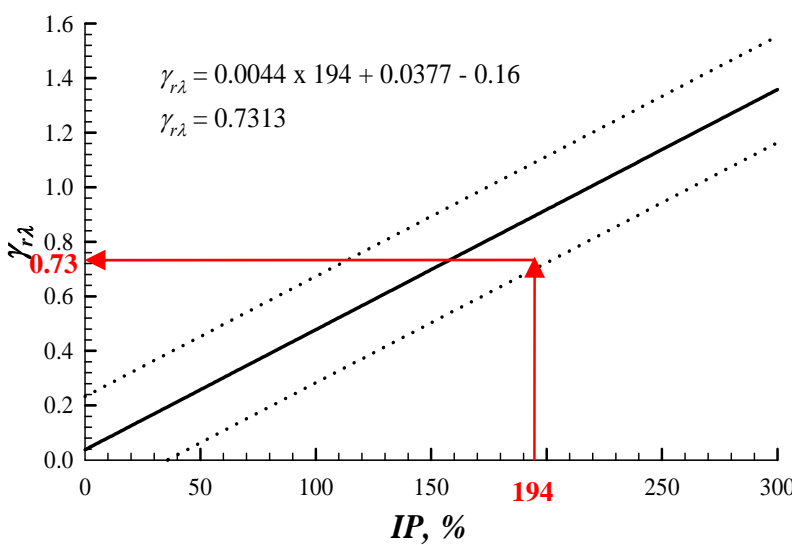

b)

Figura A-2. Obtención de los parámetros $\gamma_{r G}$ y $\gamma_{r \lambda}$.

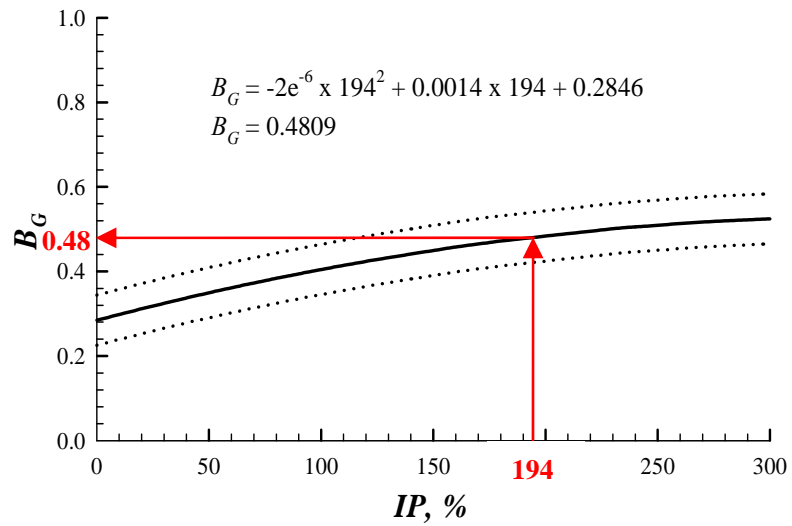

a)

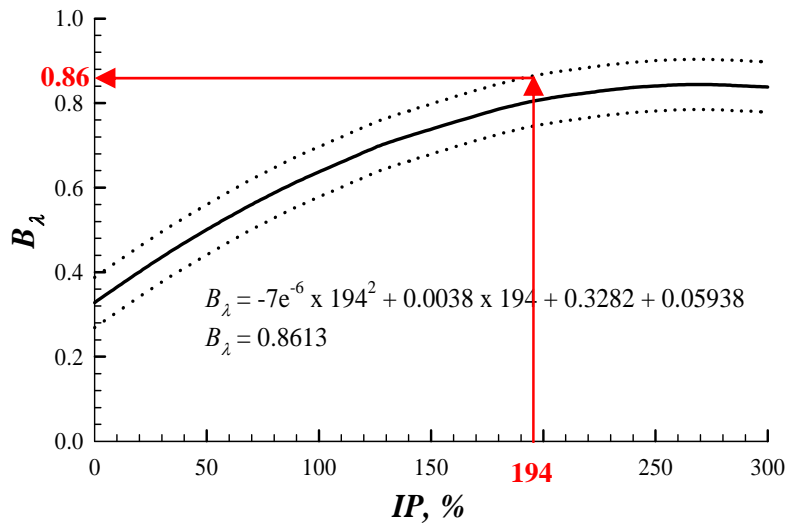

b)

Figura A-3. Obtención de los parámetros $\mathrm{B}_{G}$ y $\mathrm{B}_{\lambda}$.

$$
\begin{aligned}
& H_{G}=\frac{\left(\frac{\gamma}{\gamma_{r G}}\right)^{2 B_{G}}}{1+\left(\frac{\gamma}{\gamma_{r G}}\right)^{2 B_{G}}} \\
& H_{\lambda}=\frac{\left(\frac{\gamma}{\gamma_{r \lambda}}\right)^{2 B_{\lambda}}}{1+\left(\frac{\gamma}{\gamma_{r \lambda}}\right)^{2 B_{\lambda}}} \\
& G=\left(G_{\text {mín }}-G_{\text {máx }}\right) \cdot H_{G}+G_{\text {máx }} \\
& \lambda=\left(\lambda_{\text {máx }}-\lambda_{\text {min }}\right) \cdot H_{\lambda}+\lambda_{\text {min }}
\end{aligned}
$$


Tabla A-4. Cálculo teórico de las propiedades dinámicas.

\begin{tabular}{|c|c|c|c|c|c|c|c|c|c|}
\hline \multicolumn{5}{|c|}{ Módulo de Rígidez al Esfuerzo Cortante } & \multicolumn{5}{|c|}{ Relación de amortiguamiento } \\
\hline Columna 1 & Columna 2 & Columna 3 & Columna 4 & Columna 5 & Columna 6 & Columna 7 & Columna 8 & Columna 9 & Columna 10 \\
\hline$\gamma$ & $\gamma / \gamma_{r G}$ & $H(\gamma)$ & $G$ & $G / G_{m a ́ x}$ & $\gamma$ & $\gamma / \gamma_{r \lambda}$ & $H(\gamma)$ & $\lambda$ & $\lambda / \lambda_{\text {máx }}$ \\
\hline$\%$ & & & $\mathrm{~kg} / \mathrm{cm}^{2}$ & & $\%$ & & & $\%$ & \\
\hline 0.00040 & 0.00103 & 0.00133 & 91.64983 & 1.00000 & 0.00040 & 0.00055 & 0.00000 & 2.50003 & 0.17865 \\
\hline 0.00050 & 0.00128 & 0.00165 & 91.62075 & 0.99968 & 0.00050 & 0.00068 & 0.00000 & 2.50004 & 0.17865 \\
\hline 0.00060 & 0.00154 & 0.00197 & 91.59192 & 0.99937 & 0.00060 & 0.00082 & 0.00000 & 2.50006 & 0.17865 \\
\hline 0.00070 & 0.00180 & 0.00228 & 91.56328 & 0.99906 & 0.00070 & 0.00096 & 0.00001 & 2.50007 & 0.17865 \\
\hline 0.00080 & 0.00205 & 0.00259 & 91.53482 & 0.99875 & 0.00080 & 0.00109 & 0.00001 & 2.50009 & 0.17865 \\
\hline 0.00090 & 0.00231 & 0.00290 & 91.50651 & 0.99844 & 0.00090 & 0.00123 & 0.00001 & 2.50011 & 0.17865 \\
\hline 0.00100 & 0.00257 & 0.00321 & 91.47834 & 0.99813 & 0.00100 & 0.00137 & 0.00001 & 2.50013 & 0.17865 \\
\hline 0.00200 & 0.00513 & 0.00624 & 91.20222 & 0.99512 & 0.00200 & 0.00273 & 0.00004 & 2.50044 & 0.17868 \\
\hline 0.00300 & 0.00770 & 0.00918 & 90.93318 & 0.99218 & 0.00300 & 0.00410 & 0.00008 & 2.50089 & 0.17871 \\
\hline 0.00400 & 0.01027 & 0.01208 & 90.66916 & 0.98930 & 0.00400 & 0.00547 & 0.00013 & 2.50146 & 0.17875 \\
\hline 0.00500 & 0.01283 & 0.01493 & 90.40920 & 0.98646 & 0.00500 & 0.00684 & 0.00019 & 2.50214 & 0.17880 \\
\hline 0.00600 & 0.01540 & 0.01774 & 90.15270 & 0.98366 & 0.00600 & 0.00820 & 0.00025 & 2.50293 & 0.17885 \\
\hline 0.00800 & 0.02053 & 0.02326 & 89.64866 & 0.97816 & 0.00800 & 0.01094 & 0.00042 & 2.50481 & 0.17899 \\
\hline 0.01000 & 0.02567 & 0.02867 & 89.15500 & 0.97278 & 0.01000 & 0.01367 & 0.00061 & 2.50707 & 0.17915 \\
\hline 0.02000 & 0.05133 & 0.05436 & 86.80987 & 0.94719 & 0.02000 & 0.02735 & 0.00203 & 2.52329 & 0.18031 \\
\hline 0.04000 & 0.10266 & 0.10070 & 82.58072 & 0.90105 & 0.04000 & 0.05470 & 0.00665 & 2.57651 & 0.18411 \\
\hline 0.06000 & 0.15399 & 0.14191 & 78.81903 & 0.86000 & 0.06000 & 0.08205 & 0.01329 & 2.65282 & 0.18957 \\
\hline 0.08000 & 0.20532 & 0.17905 & 75.42931 & 0.82302 & 0.08000 & 0.10939 & 0.02163 & 2.74873 & 0.19642 \\
\hline 0.10000 & 0.25665 & 0.21279 & 72.34944 & 0.78941 & 0.10000 & 0.13674 & 0.03145 & 2.86164 & 0.20449 \\
\hline 0.11000 & 0.28232 & 0.22855 & 70.91094 & 0.77372 & 0.11000 & 0.15042 & 0.03685 & 2.92380 & 0.20893 \\
\hline 0.13500 & 0.34648 & 0.26512 & 67.57327 & 0.73730 & 0.13500 & 0.18460 & 0.05164 & 3.09382 & 0.22108 \\
\hline 0.15500 & 0.39781 & 0.29181 & 65.13786 & 0.71073 & 0.15500 & 0.21195 & 0.06461 & 3.24306 & 0.23174 \\
\hline 0.18000 & 0.46197 & 0.32239 & 62.34623 & 0.68027 & 0.18000 & 0.24614 & 0.08204 & 3.44347 & 0.24606 \\
\hline 0.20000 & 0.51330 & 0.34492 & 60.29035 & 0.65783 & 0.20000 & 0.27349 & 0.09679 & 3.61306 & 0.25818 \\
\hline 0.30000 & 0.76995 & 0.43747 & 51.84341 & 0.56567 & 0.30000 & 0.41023 & 0.17727 & 4.53860 & 0.32432 \\
\hline 0.40000 & 1.02660 & 0.50631 & 45.55958 & 0.49710 & 0.40000 & 0.54697 & 0.26127 & 5.50460 & 0.39335 \\
\hline 0.60000 & 1.53990 & 0.60235 & 36.79444 & 0.40147 & 0.60000 & 0.82046 & 0.41559 & 7.27929 & 0.52016 \\
\hline 0.80000 & 2.05320 & 0.66640 & 30.94792 & 0.33768 & 0.80000 & 1.09394 & 0.53859 & 8.69380 & 0.62124 \\
\hline 1.00000 & 2.56650 & 0.71230 & 26.75860 & 0.29197 & 1.00000 & 1.36743 & 0.63160 & 9.76336 & 0.69767 \\
\hline 2.00000 & 5.13301 & 0.82825 & 16.17556 & 0.17649 & 2.00000 & 2.73486 & 0.84981 & 12.27284 & 0.87699 \\
\hline 3.00000 & 7.69951 & 0.87689 & 11.73648 & 0.12806 & 3.00000 & 4.10228 & 0.91921 & 13.07087 & 0.93402 \\
\hline 4.00000 & 10.26601 & 0.90379 & 9.28168 & 0.10127 & 4.00000 & 5.46971 & 0.94917 & 13.41550 & 0.95865 \\
\hline 6.00000 & 15.39902 & 0.93277 & 6.63637 & 0.07241 & 6.00000 & 8.20457 & 0.97406 & 13.70168 & 0.97910 \\
\hline 8.00000 & 20.53202 & 0.94818 & 5.22993 & 0.05706 & 8.00000 & 10.93942 & 0.98403 & 13.81640 & 0.98729 \\
\hline 10.00000 & 25.66503 & 0.95776 & 4.35487 & 0.04752 & 10.00000 & 13.67428 & 0.98907 & 13.87435 & 0.99144 \\
\hline 12.00000 & 30.79803 & 0.96432 & 3.75694 & 0.04099 & 12.00000 & 16.40913 & 0.99200 & 13.90795 & 0.99384 \\
\hline 14.00000 & 35.93104 & 0.96908 & 3.32200 & 0.03625 & 14.00000 & 19.14399 & 0.99385 & 13.92928 & 0.99536 \\
\hline 16.00000 & 41.06405 & 0.97271 & 2.99114 & 0.03264 & 16.00000 & 21.87885 & 0.99511 & 13.94374 & 0.99639 \\
\hline 18.00000 & 46.19705 & 0.97556 & 2.73084 & 0.02980 & 18.00000 & 24.61370 & 0.99600 & 13.95403 & 0.99713 \\
\hline 20.00000 & 51.33006 & 0.97786 & 2.52060 & 0.02750 & 20.00000 & 27.34856 & 0.99666 & 13.96164 & 0.99767 \\
\hline 60.00000 & 153.99017 & 0.99219 & 1.21265 & 0.01323 & 60.00000 & 82.04567 & 0.99950 & 13.99420 & 1.00000 \\
\hline
\end{tabular}


Finalmente, la figura A-4 presenta gráficamente el comportamiento teórico de las propiedades dinámicas del suelo que integra el depósito de suelo supuesto. De manera adicional y a modo de validación del proceso de modelado, en la misma figura A-4 se presentan los resultados experimentales correspondientes a los teóricos, permitiendo constatar una vez más la aplicabilidad del Modelo Modificado tipo Masing para obtener el comportamiento de propiedades dinámicas de arcillas altamente plásticas.

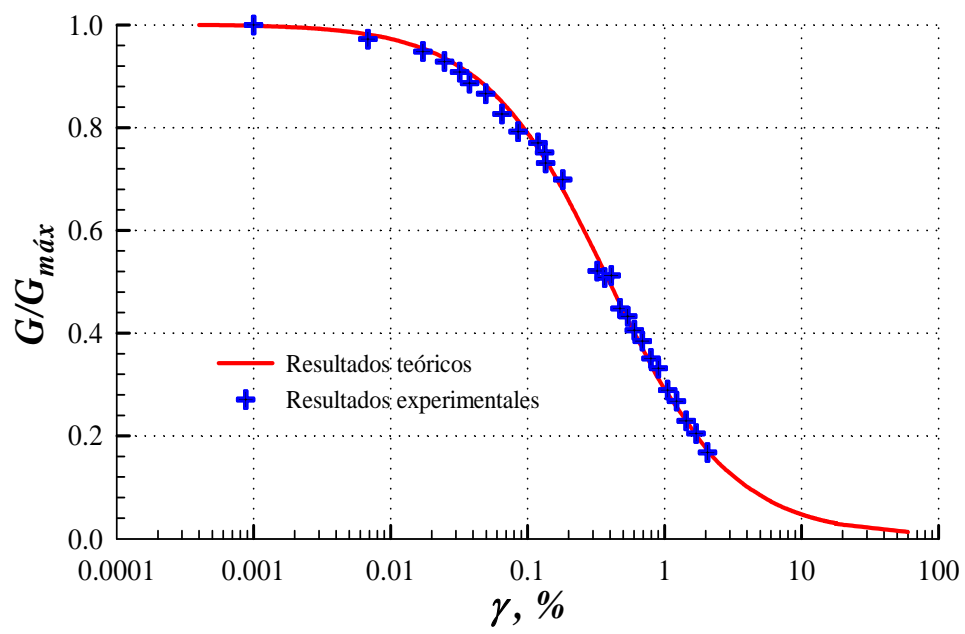

a) Relación $G$ vs $\gamma$.

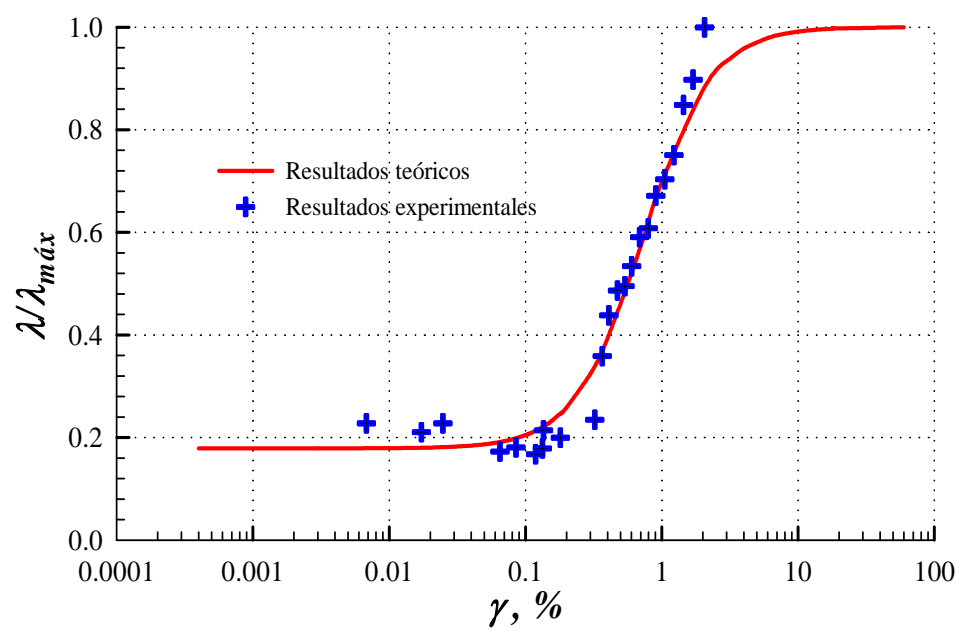

b) Relación $\lambda$ vs $\gamma$.

Figura A-4. Comportamiento de las propiedades dinámicas $G$ y $\lambda$ respecto a la $\gamma$, para un depósito de arcilla de la Ciudad de México. 